\title{
A Closed Power Allocation Solution for Outage Restricted Distributed MIMO Multi-hop Networks
}

\author{
Yidong Lang, Dirk Wübben, Carsten Bockelmann, and Karl-Dirk Kammeyer \\ Department of Communications Engineering \\ University of Bremen \\ Otto-Hahn-Allee 1, 28359 Bremen, Germany \\ Email: \{lang, wuebben, bockelmann, kammeyer\}@ant.uni-bremen.de
}

\begin{abstract}
Power consumption and Quality-of-Service are the critical factors when developing resource allocation strategies for wireless networks. In order to minimize total transmission power while meeting the end-to-end outage probability requirement in a distributed MIMO multi-hop network, we will formulate the power allocation task as a convex optimization problem. By using some approximations to the optimization problem, we derive a novel near-optimal power allocation solution with lower complexity for distributed MIMO multi-hop networks. For the network with a large number of relaying nodes per virtual antenna array even a simple closed-form solution can be obtained. The simulation results show that our solution achieves a near-optimal performance.
\end{abstract}

Index Terms-Power allocation, distributed MIMO, QoS, outage probability, relaying.

\section{INTRODUCTION}

As general acceptance, issues related to resource allocation, especially power consumption, are very important in wireless communication systems. The power consumption of wireless terminals may be one of the most limiting factors for future communication systems. On the other hand, network resources like power and bandwidth should be allocated to the nodes to support their Quality-of-Service (QoS) during their service time, e.g., low error rate or high throughput. To this end, power consumption and QoS, are the critical factors for developing suitable resource allocation strategies.

Recently, distributed multiple-input multiple-output (MIMO) techniques, which apply MIMO techniques with respect to spatially separated relaying nodes, have been shown to increase the channel capacity of wireless networks drastically [1], [2]. Fig. 1 shows the distributed MIMO transmission scheme in a wireless mesh network. Some spatially separated relaying nodes are formed into virtual antenna arrays (VAAs), which allows to increase capacity by applying distributed space-time block codes.

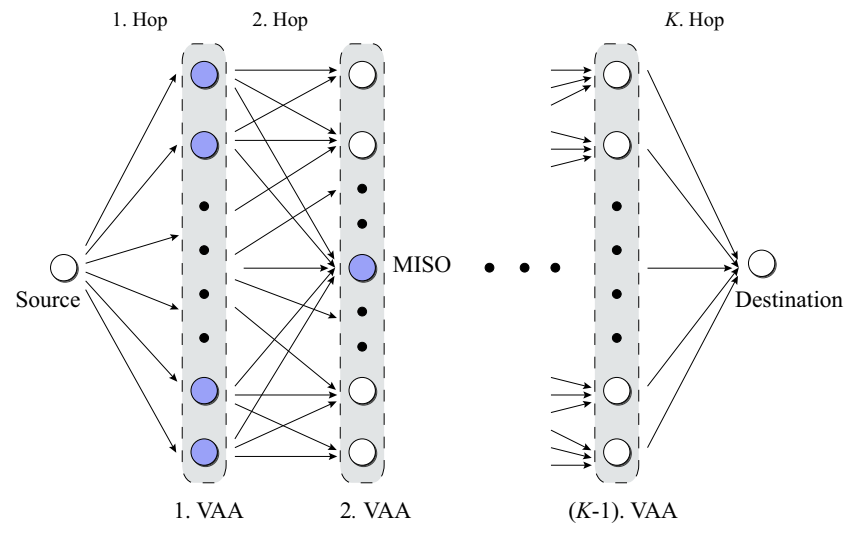

Fig. 1. System model of a distributed MIMO multi-hop system.

Two VAAs can be further composed to a distributed MIMO system, one as the virtual multiple antenna transmitter, the other as the virtual multiple antenna receiver. While the MIMO techniques that are applied to improve performance of wireless communications are well developed, e.g., [3], [4], the application of distributed MIMO in wireless mesh networks is still an open and challenging task. Especially, the end-to-end (e2e) QoS constraints like outage probability, delay, throughput as well as the power assignment per node influence the performance of the distributed MIMO multi-hop scheme.

In [1], [2], Dohler et al. have developed a resource allocation strategy for distributed MIMO multi-hop networks in order to achieve high ergodic channel capacity, where the Decode-and-Forward (D\&F) relaying protocol is used at each relays. In [5], a power allocation solution to reduce pairwise error probability (PEP) for a twohop wireless network with Amplify-and-Forward (A\&F) relaying protocol was introduced. It has been shown that the optimal power allocation assigns half the total power at the source and the other half is shared between the 
relays. In both papers a fixed total power $\mathcal{P}_{\text {total }}$ consumed in the whole network is assumed. Nevertheless, the power allocation that aims to minimize the total power consumption while satisfying an given e2e QoS constraint is much more interesting for practical systems, which will be investigated in this paper. Note that the majority of today's wireless applications happen over slow-fading channels, i.e., non-ergodic in the capacity sense, to which the concept of outage probability is applicable. Hence, it is meaningful to take the e2e outage probability as the measurement for the e2e QoS.

We consider an end-to-end transmission scenario in a wireless multi-hop network similar to [1], where D\&F is applied at topologically imposed relaying VAAs. In order to satisfy the e2e QoS requirement while reducing the resource effort, i.e., total power consumption of the whole network, the optimal power allocation strategy will be formulated as a convex optimization problem. Unfortunately, the optimization problem doesn't lead to a closed form solution. It can only be solved by timeconsuming numerical iteration with high complexity.

However, by using some approximations to the e2e outage probability expression, we will derive a nearoptimal power allocation solution for the networks with arbitrary number of nodes per VAA by solving a highorder equation with the knowledge, that the equation has a positive real solution. Furthermore, if a large number of relaying nodes per VAA is assumed, a simpler closed form solution for the power allocation can be obtained, which is the positive solution of a quadratic equation.

The remainder of the paper is organized as follows: in Section II the system model of the distributed MIMO multi-hop scheme is introduced. The mathematical description of the outage probability will be given in Section III and the optimal power allocation problem is formulated as a convex optimization problem in Section IV. A closed-form solution for the optimization problem will be derived in Section V. Finally, some simulation results and conclusions will be given in Section VI and VII, respectively.

\section{System Model}

As shown in Fig. 1, a source node desires to communicate with a destination node via $K-1$ dedicated relaying VAAs in $K$ hops. Note that each node in the network has only one antenna. We consider a time-slotted transmission scheme with half-duplex constraint at each VAA, i.e., one VAA can't transmit and receive signals simultaneously. The source broadcasts the information to the first VAA at first time slot. According to the
D\&F protocol [6], each node of the first VAA decodes the received information separately and then re-encodes the decoded information "cooperatively" according to a space-time code word. Each node use only a spatial fraction of the space-time code word. At the second time slot the first VAA transmits information to the second VAA. Each node of the second VAA decodes the information separately, re-encodes, and retransmits it to the next VAA in the same manner as in the first time slot. The information is passed from one VAA to another VAA until it reaches the destination. Mention that if one VAA is far away from another VAA, both VAAs can transmit information at the same time slot without interference. As the information is transmitted from the source node through a number of hops to the destination node, the network we investigated here is often referred to as a distributed MIMO multi-hop network. The focus of this paper is on the power allocation strategy for the network, thus, for the further investigation a given fixed network topology is assumed, i.e. the task of forming the VAAs is not considered here.

As described above, the nodes within the same VAA decode the information separately but re-encode the decoded information by using a spatial fraction of the space-time code word. Therefore, the transmission within one hop can be modeled as several multipleinput single output (MISO) systems. We assume that each VAA transmits signals with the same rate $R$ and all the hops use the total bandwidth $W$ that is available to the network. We let $k$ index the hop, $t_{k}, r_{k}$ denote the number of transmit nodes and receive nodes within the $k$ th hop, respectively. We define $\mathbf{S}_{k} \in \mathbb{C}^{t_{k} \times T}$ as the space-time encoded signal with length $T$ from the $t_{k}$ nodes at the $k$ th hop. The received signal $\mathbf{y}_{k, j} \in \mathbb{C}^{1 \times T}$ at the $j$ th node at the $k$ th VAA is given by

$$
\mathbf{y}_{k, j}=\sqrt{\frac{\gamma_{k} \mathcal{P}_{k}}{t_{k}}} \mathbf{h}_{k, j} \mathbf{S}_{k}+\mathbf{n}_{k, j}
$$

where $\mathbf{n}_{k, j} \sim \mathcal{N}_{C}\left(0, N_{0}\right) \in \mathbb{C}^{1 \times T}$ denotes the Gaussian noise vector with power spectral density $N_{0}$ and $\mathcal{P}_{k}$ is the total power of the $k$ th hop. The channel from the $t_{k}$ transmit nodes to the $j$ th receive node within the $k$ th hop is expressed as $\mathbf{h}_{k, j} \in \mathbb{C}^{1 \times t_{k}}$. The elements of $\mathbf{h}_{k, j}$ obey the same uncorrelated Rayleigh fading statistics, i.e. complex zero-mean circular symmetric Gaussian distribution with variance 1 . We assume that the relaying nodes belonging to the same VAA are spatially sufficiently close as to justify a common pathloss $\gamma_{k}$ between two VAAs. It is described as $\gamma_{k}=\left(\frac{1}{d_{k}}\right)^{\epsilon}$, where $d_{k}$ is the distance between the transmit nodes and the receive 
nodes at the $k$ th hop and $\epsilon$ is the pathloss exponent within range of 2 to 5 for most wireless channels.

\section{Outage Probability}

According to the capacity of a MIMO channel exposed in [3], [4], the capacity of a MISO system described in (1) is

$$
C_{k, j}=W \log \left(1+\frac{\mathcal{P}_{k}}{t_{k} d_{k}^{\epsilon} W N_{0}}\left\|\mathbf{h}_{k, j}\right\|^{2}\right)
$$

with the $l_{2}$-norm, i.e., $\left\|\mathbf{h}_{k, j}\right\|^{2}=\sum_{i=1}^{t_{k}}\left|h_{k, i, j}\right|^{2}$. In the sequel we investigate the outage probability $P_{\text {out }, k, j}$ of the $j$ th receiving node at the $k$ th hop, which defines the probability that the transmit rate $R$ is greater than the instantaneous channel capacity $C_{k, j}$. It is expressed as a cumulative distribution function (CDF), which depends on the channel condition within the hop, the power $P_{k}$ and bandwidth $W$ spent on it, and the data rate $R$,

$$
\begin{aligned}
P_{\text {out }, k, j} & =\operatorname{Pr}\left(R>C_{k, j}\right) \\
& =\operatorname{Pr}\left(R>W \log \left(1+\frac{\mathcal{P}_{k}}{t_{k} d_{k}^{\epsilon} W N_{0}}\left\|\mathbf{h}_{k, j}\right\|^{2}\right)\right) \\
& =\operatorname{Pr}(\left\|\mathbf{h}_{k, j}\right\|^{2}<\underbrace{\frac{\left(2^{\frac{R}{W}}-1\right) W N_{0} d_{k}^{\epsilon} t_{k}}{\mathcal{P}_{k}}}_{x_{k}}) \\
& =\frac{\gamma\left(t_{k}, x_{k}\right)}{\Gamma\left(t_{k}\right)} .
\end{aligned}
$$

For simplicity we define $x_{k}=\left(2^{\frac{R}{W}}-1\right) W N_{0} d_{k}^{\epsilon} t_{k} / \mathcal{P}_{k}$, which is proportional to the noise-to-signal ratio, i.e., 1/SNR. Herein, $\left\|\mathbf{h}_{k, j}\right\|^{2}$ obeys a Gamma distribution [7], therefore its CDF can be described by a incomplete Gamma function $\gamma\left(t_{k}, x_{k}\right)=\int_{0}^{x_{k}} e^{-u} u^{t_{k}-1} \mathrm{~d} u$.

As mentioned before a common pathloss $\gamma_{k}$ within a hop is assumed. Thus, each MISO system within one hop has the same outage probability

$$
P_{\text {out }, k, 1}=P_{\text {out }, k, 2}=\cdots=P_{\text {out }, k, r_{k}} .
$$

Then the outage probability within the $k$ th hop is given by

$P_{\mathrm{out}, k}=1-\prod_{j=1}^{r_{k}}\left(1-P_{\mathrm{out}, k, j}\right)=1-\left(1-P_{\mathrm{out}, k, j^{\prime}}\right)^{r_{k}}$,

where $j^{\prime}$ indexes an arbitrary $j \in\left[1, \cdots, r_{k}\right]$. Since D\&F is applied, the signals are completely decoded at each VAA, so that the outage probabilities per hop $P_{\text {out }, k}, \forall k$ are mutually independent. Thus, the end-to-end outage probability is given by

$$
P_{\mathrm{e} 2 \mathrm{e}}=1-\prod_{k=1}^{K}\left(1-P_{\mathrm{out}, k}\right)=1-\prod_{k=1}^{K}\left(1-P_{\mathrm{out}, k, j^{\prime}}\right)^{r_{k}},
$$

where (5) was used. Note that for simplicity, we assume that the e2e communication is in outage if any of the MISO systems can't correctly decode the information. In the following investigation we use the end-to-end outage probability $P_{\mathrm{e} 2 \mathrm{e}}$ as the measurement for the required QoS.

\section{Optimization Problem}

The optimization problem that minimizes the total power of the whole network while meeting the end-toend outage probability requirement $e$ can be formulated as follows

$$
\begin{aligned}
& \text { minimize } \mathcal{P}_{\text {total }}=\sum_{k=1}^{K} \mathcal{P}_{k} \\
& \text { subject to } P_{\text {e2e }} \leq e .
\end{aligned}
$$

Note that (7) can be proven to be convex for low outage probability constraint $e$ by proving the Hessian matrix of $P_{\mathrm{e} 2 \mathrm{e}}\left(\mathcal{P}_{k}, \forall k\right)$ to be positive semi-definite [8]. Unfortunately, even for the simplest models of the outage probability, the optimization problem (7) doesn't lead to a closed-form solution in terms of the power per hop, due to the complex product description of the end-toend outage probability (6). However, it can be solved by standard optimization tools leading to considerable complexity.

\section{Closed Power Allocation Solution}

In the sequel, some approximations for the optimization problem (7) are introduced in order to derive a nearoptimal power allocation solution.

As the low outage probability region is concerned for practical systems, a simple approximated expression of the outage probability will be used [9]. The incomplete Gamma function $\gamma\left(t_{k}, x_{k}\right)$ can be described by the confluent hypergeometric function ${ }_{1} F_{1}(\cdot)[10]$ pp. 262

$$
\gamma\left(t_{k}, x_{k}\right)=t_{k}^{-1} x_{k}^{t_{k}} F_{1}\left(t_{k}, 1+t_{k},-x_{k}\right) .
$$

For the low outage probability region (e.g., $e=1 \%$ ), we have $x_{k} \rightarrow 0$, thereby the confluent hypergeometric function can be approximated by [10] pp. 504

$$
\begin{aligned}
{ }_{1} F_{1}\left(t_{k}, 1+t_{k},-x_{k}\right) & =1-\sum_{m=1}^{\infty}(-1)^{m} \frac{t_{k}}{\left(t_{k}+m\right) m !} x_{k}^{m} \\
& \leq 1 .
\end{aligned}
$$


Therefore, we achieve a simple approximation for (3)

$$
\begin{aligned}
P_{\mathrm{out}, k, j} & =\frac{\gamma\left(t_{k}, x_{k}\right)}{\Gamma\left(t_{k}\right)}=\frac{t_{k}^{-1} x_{k}^{t_{k}}{ }_{1} F_{1}\left(t_{k}, 1+t_{k},-x_{k}\right)}{\Gamma\left(t_{k}\right)} \\
& \approx \frac{t_{k}^{-1} x_{k}^{t_{k}}}{\Gamma\left(t_{k}\right)}=\frac{x_{k}^{t_{k}}}{\Gamma\left(t_{k}+1\right)} .
\end{aligned}
$$

Similar to the approximation used in [11], we can further approximate the product representation of the outage probability to a sum expression. Thus, the outage probability per hop (5) is expressed as

$$
P_{\mathrm{out}, k} \approx \sum_{j=1}^{r_{k}} P_{\mathrm{out}, k, j}=r_{k} P_{\mathrm{out}, k, j^{\prime}}=\frac{r_{k} x_{k}^{t_{k}}}{\Gamma\left(t_{k}+1\right)} .
$$

Moreover the end-to-end outage probability (6) is approximated in the same way

$$
P_{\mathrm{e} 2 \mathrm{e}} \approx \sum_{k=1}^{K} P_{\mathrm{out}, k} \approx \sum_{k=1}^{K} r_{k} P_{\mathrm{out}, k, j^{\prime}} .
$$

Comparing the approximated form (12) to the exact form (6), it has been proven in [11] that

$$
P_{\text {e2e }} \leq P_{\text {e2e, app }}=\sum_{k=1}^{K} r_{k} P_{\text {out }, k, j^{\prime}} .
$$

Thus, if we use the value of $P_{\text {e2e,app }}$ to describe $P_{\text {e2e }}$, the end-to-end outage probability constraint is more stringent. In other words, if the power is allocated to the nodes to satisfy $P_{\text {e2e,app }} \leq e$, then $P_{\text {e2e }} \leq e$ is automatically fulfilled. Inserting (13) in the optimization problem (7), we have

$$
\begin{aligned}
& \text { minimize } \mathcal{P}_{\text {total }}=\sum_{k=1}^{K} \mathcal{P}_{k} \\
& \text { subject to } P_{\text {e2e, app }} \leq e .
\end{aligned}
$$

Note that the optimization problem (14) only leads to a near-optimal power allocation solution. However, from the complexity point of view, it is reasonable to use this relatively simple form to derive a closed form solution.

The near-optimal solution can be readily obtained by solving the constrained optimization problem (14) using Lagrange multipliers. Since the e2e outage probability is the monotonous decreasing function of the power $\mathcal{P}_{k}, \forall k$, the optimal power allocation is attained when $P_{\text {e2e,app }}=e$. We define the Lagrangian as $L\left(\mathcal{P}_{k}, \lambda\right)=$ $\sum_{k=1}^{K} \mathcal{P}_{k}+\lambda\left(P_{\text {e2e,app }}-e\right)$. Hence, the near-optimal power allocation solutions $P_{k}^{*}$ satisfy

$$
\begin{gathered}
\frac{\partial L\left(\mathcal{P}_{k}, \lambda\right)}{\partial \mathcal{P}_{k}}=1+\lambda \frac{\partial P_{\text {out }, k}}{\partial \mathcal{P}_{k}}=0, \quad \forall k \\
P_{\text {e2e,app }}\left(\mathcal{P}_{k}^{*}\right)=\sum_{k=1}^{K} r_{k} P_{\text {out }, k, j^{\prime}}\left(\mathcal{P}_{k}^{*}\right)=e .
\end{gathered}
$$

Theorem 1 (Closed power allocation solution): Under the approximations (9), (13), an arbitrary number of nodes $t_{k}$ per VAA and a given e2e outage probability requirement $e$, the near-optimal power allocation $\mathcal{P}_{k}^{*}$ is given by

$$
\mathcal{P}_{k}^{*}=\left(2^{\frac{R}{W}}-1\right) W N_{0} d_{k}^{\epsilon} t_{k}\left(\frac{P_{\text {out }, k}^{*} \Gamma\left(t_{k}+1\right)}{r_{k}}\right)^{-\frac{1}{t_{k}}},
$$

with outage probability

$$
\begin{gathered}
P_{\text {out }, 1}^{*}=A^{\frac{1}{2}} \cdot a_{1} \quad k=1, \\
P_{\text {out }, k}^{*}=A^{\frac{t_{k}}{t_{k}+1}} \cdot a_{k} \quad k \geq 2,
\end{gathered}
$$

where $A$ satisfies the following high-order equation

$$
e=\sum_{k=1}^{K} P_{\text {out }, k}=\sum_{k=1}^{K} r_{k}\left(\frac{A d_{k}^{\epsilon}}{r_{k} \Gamma\left(t_{k}+1\right)^{\frac{1}{t_{k}}}}\right)^{\frac{t_{k}}{t_{k}+1}} .
$$

For the case with a large number of relaying nodes per VAA, i.e., $t \rightarrow \infty$, the closed form of $A$ is given by

$$
\begin{aligned}
A^{\frac{1}{2}} & =\frac{-a_{1}+\sqrt{a_{1}^{2}+4 e \sum_{k=2}^{K} a_{k}}}{2 \sum_{k=2}^{K} a_{k}}, \\
a_{1} & =\left(r_{1} d_{1}^{\epsilon}\right)^{\frac{1}{2}} \quad k=1, \\
a_{k} & =\left(\frac{d_{k}^{t_{k} \epsilon}}{\Gamma\left(t_{k}+1\right)}\right)^{\frac{1}{t_{k}}} \quad k \geq 2 .
\end{aligned}
$$

\section{Proof: See Appendix.}

In the sequel, we have the following observations.

Observation 1: (Near-optimal allocation of outage probability per hop:)

As it will be proven in the appendix that $0<A \ll 1$, then $A^{\frac{1}{2}} \gg A$. According to (17a) and (17b), it can seen that $P_{\text {out }, 1}^{*} \gg P_{\text {out }, k}^{*}, k \geq 2$. In other words, due to the lack of diversity gain at the first hop, the near-optimal outage probability requirement should be much looser than the outage probability requirement of the other hops with high diversity degrees.

Observation 2: (Independence of near-optimal outage probability per hop:)

From (17a) and (17b), the near-optimal outage probability per hop $P_{\text {out }, k}^{*}$ is independent from the data rate $R$ and the bandwidth $W$. If the network configuration (e.g., $\left.t_{k}, r_{k}, K\right)$ is fixed and the end-to-end outage probability is upper bounded, the near-optimal outage probabilities are also fixed. 


\section{PERFORMANCE}

The performance of the proposed closed solution for power allocation is assessed here for various network configurations. For simplicity, we consider the distributed MIMO multi-hop network with $K=5$ hops and the same number of relaying nodes $t=t_{k}, k=$ $2, \cdots, K$. Three cases is considered, i.e., $t=5, t=10$, $t=20$ number of relaying nodes per VAA. We assume that the e2e communication over $W=5 \mathrm{MHz}$ should meet an e2e outage probability constraint $e=1 \%$. The distances between each VAA are $\mathbf{d}=[1,1,1,1,1] \mathrm{km}$ and the pathloss exponent $\epsilon=3$. Furthermore we assume $N_{0}=-174 \mathrm{dBm}$ according to the UMTS standards. Fig. 2 depicts the optimal total transmission power obtained via numerical optimization of (7) and the nearoptimal total power consumption by the closed solution (1) versus the data rate $R$ in bps. It can be observed that the developed solution achieves almost the same performance as the optimal one, especially for a large number of relaying nodes per VAA $(t=10, t=20)$. For a small number of relaying nodes $(t=5)$, it only leads to a slightly higher power usage. The reason for this is that we use the more stringent $P_{\text {e2e,app }}$ instead of the exact e2e outage probability of the system $P_{\mathrm{e} 2 \mathrm{e}}$, which causes higher power consumption but meanwhile satisfies the exact e2e outage probability requirement for sure.

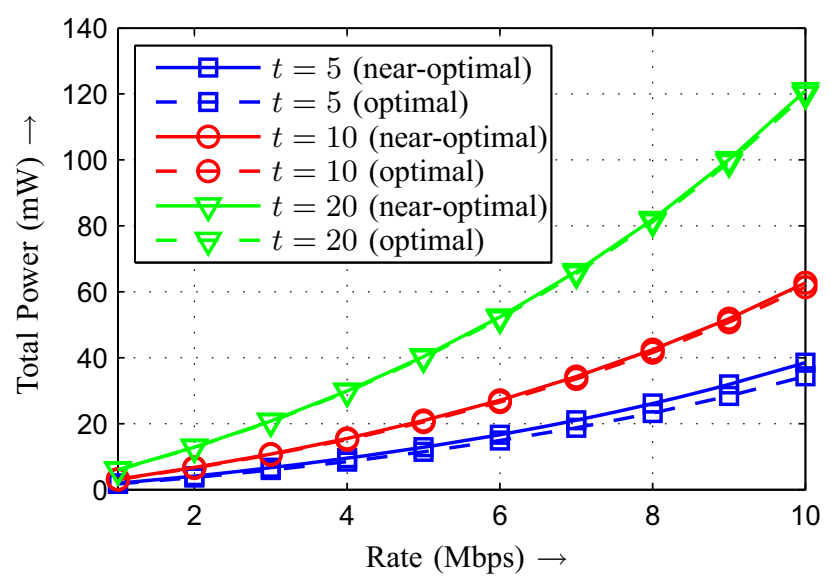

Fig. 2. Total power consumption for optimal and near-optimal power allocation. (--) denotes the optimal solution, (-) is the proposed closed form solution.

Fig. 3 shows the optimal and near-optimal power allocation $\mathcal{P}_{k}$ per hop versus rate with respect to the total power consumption $\mathcal{P}_{\text {total }}$, where $t=5$. In order to illustrate the results clearly, we choose $\log \frac{\mathcal{P}_{k}}{\mathcal{P}_{\text {total }}}$. From the figure, we have the following observation.
Observation 3: (Near-optimal power allocation per hop)

The closed-form solution achieve near performance of the optimal solution. Similar to Observation 1, most of the power should be allocated to the first hop, i.e., $\mathcal{P}_{1} \gg \mathcal{P}_{k}, k \geq 2$. Consequently, if one source communicates with its destination, the source consumes the most power, whereas the relaying nodes only require only a small fraction of the total power, but can lead to a significant performance improvement for the end-to-end communication. Hence, the transmission of the first hop is the bottleneck of the end-to-end communication.
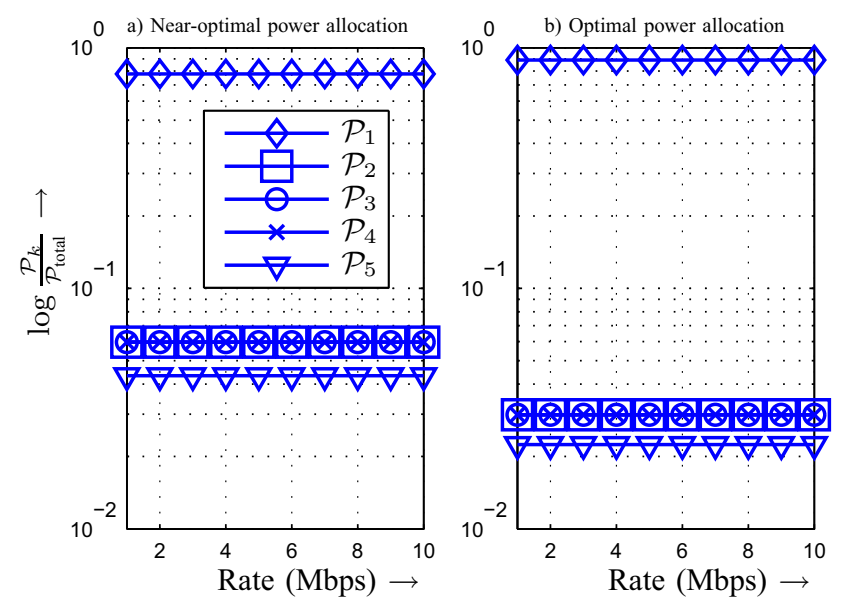

Fig. 3. Optimal and near-optimal power allocation per hop vs. rate with $t=5$.

\section{CONCLUSION}

In this paper, we analyzed power allocation strategies for e2e outage probability restricted distributed MIMO multi-hop networks with Decode-and-Forward relaying protocol. A convex optimization problem that aims to reduce the total power cost while meeting an e2e outage level was formulated, which can be solved by numerical optimization with standard optimization tools. In order to derive a simple power allocation solution with lower complexity, some reasonable approximations for the e2e outage probability were used. We proposed an efficient power allocation strategy for the network with an arbitrary number of relaying nodes per VAA. For the case of large number of relaying nodes per VAA, even a closedform solution can be achieved. The simulation results have shown that our new solution achieves near-optimal performance at low computational cost.

As shown in [5], for a distributed MIMO $K=2$-hop network with Amplify-and-Forward relaying protocol, the optimal power allocation is such that half the total 
power is used at the source and the other half is shared in the first VAA. In contrast, for the Decode-and-Forward case, we have shown that due to the lack of diversity most of the power and the outage probability should be "allocated" at the first hop, i.e., the source node uses the largest part of the total transmission power (e.g., about $90 \%$ in our case), whereas the other hops with higher diversity degrees use only a small fraction of the total power to achieve low outage probability.

\section{APPENDIX}

Proof of Theorem 1: Principally, the near-optimal solution is obtained by solving (15). For simplicity, we define $Q=\left(2^{\frac{R}{W}}-1\right) W N_{0}$, then $x_{k}=Q d_{k}^{\epsilon} t_{k} / \mathcal{P}_{k}$ follows from (3) and the first derivation of the approximated $P_{\text {out }, k}(11)$ is given by

$$
\begin{aligned}
\frac{\partial P_{\text {out }, k}}{\partial \mathcal{P}_{k}} & =\frac{\partial\left(\frac{r_{k} x_{k}^{t_{k}}}{\Gamma\left(t_{k}+1\right)}\right)}{\partial \mathcal{P}_{k}}=\frac{r_{k} t_{k} x_{k}^{t_{k}-1}}{\Gamma\left(t_{k}+1\right)} \cdot \frac{\partial x_{k}}{\partial \mathcal{P}_{k}} \\
& =-\frac{r_{k} t_{k} x_{k}^{t_{k}-1}}{\Gamma\left(t_{k}+1\right)} \cdot \frac{x_{k}}{\mathcal{P}_{k}}=-\frac{r_{k} x_{k}^{t_{k}+1}}{\Gamma\left(t_{k}+1\right) Q d_{k}^{\epsilon}} \\
& =-\frac{r_{k} P_{\text {out }, k, j^{\prime}}^{\frac{t_{k}+1}{t_{k}}} \Gamma\left(t_{k}+1\right)^{\frac{1}{t_{k}}}}{Q d_{k}^{\epsilon}}
\end{aligned}
$$

From (15a) we know that the first derivatives of the outage probabilities with respect to each power allocation are all the same

$$
\frac{\partial P_{\text {out }, 1}}{\partial \mathcal{P}_{1}}=\frac{\partial P_{\text {out }, 2}}{\partial \mathcal{P}_{2}}=\cdots=\frac{\partial P_{\text {out }, K}}{\partial \mathcal{P}_{K}},
$$

thus we define constant $A$ as

$$
\frac{\partial P_{\mathrm{out}, k}}{\partial \mathcal{P}_{k}}=\frac{r_{k} P_{\mathrm{out}, k, j^{\prime}}^{\frac{t_{k}+1}{t_{k}}} \Gamma\left(t_{k}+1\right)^{\frac{1}{t_{k}}}}{d_{k}^{\epsilon}}=A, \quad \forall k .
$$

As the lower region of the outage probability and long distance $d_{k}=1000 \mathrm{~m}$ are considered, $0<A \ll 1$ follows. Rewriting (22) and inserting into (11), $P_{\text {out }, k}$ is described by

$$
P_{\text {out }, k}=r_{k} P_{\text {out }, k, j^{\prime}}=r_{k}\left(\frac{A d_{k}^{\epsilon}}{r_{k} \Gamma\left(t_{k}+1\right)^{\frac{1}{t_{k}}}}\right)^{\frac{t_{k}}{t_{k}+1}} .
$$

Inserting (23) into the equation (15b) we achieve

$$
e=\sum_{k=1}^{K} P_{\mathrm{out}, k}=\sum_{k=1}^{K} r_{k}\left(\frac{A d_{k}^{\epsilon}}{r_{k} \Gamma\left(t_{k}+1\right)^{\frac{1}{t_{k}}}}\right)^{\frac{t_{k}}{t_{k}+1}} .
$$

Note that here the solution of the high-order equation (24) is applicable for an arbitrary number of relaying nodes per VAA. Since $A$ is real and positive, the equation can be solved analytically. For the case of large $t_{k}$, even a simple closed-form solution can be obtained.

For $k=1, t_{k}=1$ (with $\Gamma(2)=1$ ), (23) is wrote as

$P_{\text {out }, 1}=A^{\frac{1}{2}}\left(r_{1} d_{1}^{\epsilon}\right)^{\frac{1}{2}}=A^{\frac{1}{2}} a_{1}$, with $a_{1}=\left(r_{1} d_{1}^{\epsilon}\right)^{\frac{1}{2}}$.

For $k \geq 2$, large $t_{k}$, by applying $\frac{t_{k}}{t_{k}+1} \approx 1$, (23) can be simplified as

$P_{\mathrm{out}, k} \approx \frac{A d_{k}^{\epsilon}}{\Gamma\left(t_{k}+1\right)^{\frac{1}{t_{k}}}}=A a_{k}$, with $a_{k}=\frac{d_{k}^{\epsilon}}{\Gamma\left(t_{k}+1\right)^{\frac{1}{t_{k}}}}$.

Inserting (25), (26) to (24) we achieve

$$
e \approx A^{\frac{1}{2}} \cdot a_{1}+A \cdot \sum_{k=2}^{K} a_{k} .
$$

By solving (27) we have

$$
A^{\frac{1}{2}}=\frac{-a_{1}+\sqrt{a_{1}^{2}+4 e \sum_{k=2}^{K} a_{k}}}{2 \sum_{k=2}^{K} a_{k}} .
$$

Inserting (28) to (25), (26), the closed form solutions (17a), (17b) are obtained. The near-optimal power allocation $\mathcal{P}_{k}^{*}(16)$ can be then directly derived from (11).

\section{REFERENCES}

[1] M. Dohler, A. Gkelias, and H. Aghvami, "A Resource Allocation Strategy for Distributed MIMO Multi-Hop Commonication Systems," IEEE Communications Letters, vol. 8, no. 2, pp. 98 101, February 2004.

[2] M. Dohler, Virtual Antenna Arrays, Ph.D. thesis, King's College London, U.K., November 2003.

[3] I. Telatar, "Capacity of Multi-antenna Gaussian Channels," European Transactions on Telecommunications, vol. 10, no. 6 , pp. 585-595, November/December 1999.

[4] E.G. Larsson and P. Stoica, Space-Time Block Coding for Wireless Communications, Cambridge University Press, 2003.

[5] Y. Jing and B. Hassibi, "Distributed Space-Time Coding in Wireless Relay Networks," IEEE Trans. on Wireless Communications, vol. 5, no. 12, pp. 3524-3536, December 2006.

[6] J. N. Laneman, D. Tse, and G. Wornell, "Cooperative Diversity in Wireless Networks: Efficient Protocols and Outage Behavior," IEEE Trans. on Information Theory, vol. 50, no. 12, pp. 3062-3080, February 2004.

[7] M. Kendall and A. Stuart, The Advanced Theory of Statistics, vol. 1, Griffen, London, U.K., 4. edition, 1979.

[8] Y. Lang, "Resource Allocation for Distributed MIMO in Wireless Networks," Diploma Thesis, Department of Communications Engineering, University of Bremen, September 2007.

[9] M. Dohler and M. Arndt, "Inverse Incomplete Gamma Function and Its Application," IEE Electronics Letters, vol. 42, no. 1, pp. 35-6, January 2006.

[10] M. Abramowitz and I. Stegun, Handbook of Mathematical Functions with Formulas, Graphs, and Mathematical Tables, Dover Publications, 1970.

[11] R. Manohar and A. Scaglione, "Power Optimal Routing in Wireless Networks," Techincal Report CSL-TR-2003-1028, Computer Systems Laboratory, Cornell University, 2003. 